\title{
Stroke Increases G Protein-Coupled Estrogen Receptor Expression in the Brain of Male but Not Female Mice
}

\author{
Brad R.S. Broughton ${ }^{a}$ Vanessa H. Brait ${ }^{a}$ Elizabeth Guida ${ }^{a}$ Seyoung Lee ${ }^{a}$ \\ Thiruma V. Arumugam ${ }^{b}$ Chantelle V. Gardiner-Mann ${ }^{a} \quad$ Alyson A. Miller $^{a}$ \\ Sung-Chun Tang ${ }^{c}$ Grant R. Drummond ${ }^{a}$ Christopher G. Sobey ${ }^{\mathrm{a}}$ \\ a Vascular Biology and Immunopharmacology Group, Department of Pharmacology, Monash University, Clayton, \\ Vic., 'b School of Biomedical Sciences, University of Queensland, Brisbane, Qld., Australia; 'Department of \\ Neurology and Stroke Center and Department of Pathology, National Taiwan University Hospital and National \\ Taiwan University College of Medicine, Taipei, Taiwan, ROC
}

\section{Key Words}

GPER - Cerebral ischemia - Sex specific - Infarct core •

Peri-infarct region $\cdot$ Human $\cdot$ Middle cerebral artery occlusion

\begin{abstract}
The novel estrogen receptor, $G$ protein-coupled estrogen receptor (GPER, previously named GPR30), is widely distributed throughout the male and female brain and, thus, could potentially play a role in estrogen-mediated neuroprotective effects in diseases such as stroke. We hypothesized that GPER distribution and expression in the brain of male, intact female, and ovariectomized (OVX) mice is increased after 0.5 $\mathrm{h}$ middle cerebral artery occlusion. Using immunohistochemistry, we found that ischemia reperfusion increased GPER distribution in the peri-infarct brain regions of male mice, but surprisingly not in intact females or OVX mice. Similar differences were observed in the male and female human brain after stroke. In contrast, GPER distribution was decreased in the infarct core of all mice examined. Furthermore, GPER immunofluorescence was co-localized with the endothelial cell marker, von Willebrand factor, and the neu-
\end{abstract}

\begin{tabular}{ll}
\hline KARGER & $\begin{array}{l}\text { ( } 2012 \text { S. Karger AG, Basel } \\
1424-862 X / 13 / 0214-0229 \$ 38.00 / 0 \quad \text { Karger }\end{array}$ \\
E-Mail karger@karger.com & $\begin{array}{l}\text { This is an Open Access article licensed under the terms of the } \\
\text { www.karger.com/nsg }\end{array}$ \\
& $\begin{array}{l}\text { Creative Commons Attribution-NonCommercial 3.0 Un- } \\
\text { ported license (CC BY-NC) (www.karger.com/OA-license), } \\
\text { applicable to the online version of the article only. Distribu- } \\
\text { tion permitted for non-commercial purposes only. }\end{array}$
\end{tabular}

ronal marker, NeuN. Consistent with the immunohistochemical findings, Western blot analysis showed GPER expression is only elevated in the ischemic hemisphere of male mice. Moreover, GPER mRNA expression in males was elevated at $4 \mathrm{~h}$ but had returned to baseline by $24 \mathrm{~h}$. In conclusion, these findings indicate that GPER may be a potential therapeutic target after stroke, especially in males, in whom estrogen therapy is not feasible.

Copyright @ 2012 S. Karger AG, Basel

\section{Introduction}

Traditionally, estrogen was considered to mediate its actions solely via activation of the classical nuclear receptors, estrogen receptors $\alpha$ and $\beta$. However, studies have now identified a novel estrogen receptor called $G$ proteincoupled estrogen receptor (GPER, previously named GPR30) $[1,2]$. This former orphan receptor belongs to the family of seven transmembrane $G$ protein-coupled recep-

B.R.S.B. and V.H.B. are equal first authors.
Brad R.S. Broughton, $\mathrm{PhD}$

Department of Pharmacology

Monash University

Clayton, VIC 3800 (Australia)

Tel. +61 39905 0915, E-Mail bradley.broughton@monash.edu 
tors and binds to estradiol (E2) with high affinity and potency, thus leading to the unique classification of GPER as a membrane-localized estrogen receptor [3]. GPER expression has since been identified in tissues of several physiological systems, including the nervous, immune, cardiovascular and reproductive systems, and it is postulated to also play varied roles in cell growth and cancer, and metabolism and obesity [4].

Several studies have demonstrated that GPER is widely distributed throughout the central nervous system [5-8]. More specifically, high GPER expression has been identified in numerous regions of the brain, including the striatum, hippocampus, anterior and posterior pituitary, and granule cells of the dentate gyrus $[6,9]$, which is a profile distinct from that of the traditional estrogen receptors [5, 7]. In addition, we have reported that GPER expression is localized throughout the cerebral arterial wall in male and female rats [10]. Due to its high expression and extensive distribution in the brain and cerebral vasculature, GPER has been proposed as a possible therapeutic target for neurological diseases, including stroke. To date, only two studies have assessed the role of GPER signaling following stroke. A preliminary study of GPER signaling by Zhang et al. [11] demonstrated that chronic pretreatment of ovariectomized (OVX) mice with the GPER agonist, G-1, reduces infarct volume at $96 \mathrm{~h}$ after stroke in the striatum, cortex, and total hemisphere. More recently, Lebesgue et al. [12] showed that intracerebroventricular administration of G-1 at the time of reperfusion improved the number of surviving CA1 pyramidal neurons in young and middle-aged OVX rats following global cerebral ischemia compared with vehicle treatment. Subsequently, both Zhang et al. [11] and Lebesgue et al. [12] suggested GPER to be an attractive candidate for therapeutic intervention against ischemic brain injury. However, nothing is yet known about the effect of stroke on GPER expression. We thus hypothesized that GPER expression in the brain is upregulated following 0.5-hour middle cerebral artery (MCA) occlusion. To address this hypothesis, we assessed GPER distribution and expression in the brains of male, female, and OVX mice at $24 \mathrm{~h}$ after stroke using immunohistochemical, Western blot, and PCR analysis.

\section{Methods}

\section{Animals}

This study was conducted in accordance with National Health and Medical Research Council of Australia guidelines for the care and use of animals in research. A total of 84 mice were studied, consisting of 8 - to 12 -week-old males ( $\mathrm{n}=37$; weight: $25.6 \pm$
$0.4 \mathrm{~g})$ and females $(\mathrm{n}=47$; weight: $21.2 \pm 0.2 \mathrm{~g})$. Analyses of infarct volume and immunohistochemistry were performed on sections from the same groups of mice (male sham: $\mathrm{n}=7$; female sham: $\mathrm{n}=6$; OVX: $\mathrm{n}=6$; male stroke: $\mathrm{n}=7$; female stroke: $\mathrm{n}=6$; OVX stroke: $n=6$ ). By contrast, analyses of protein expression by Western blotting were performed in brains from separate animals (male sham: $\mathrm{n}=5$; female sham: $\mathrm{n}=5$; OVX: $\mathrm{n}=5$; male stroke: $\mathrm{n}=5$; female stroke: $\mathrm{n}=5$; OVX stroke: $\mathrm{n}=5$ ). Moreover, PCR experiments were performed in separate animals (male sham: $\mathrm{n}=3$; male stroke: $\mathrm{n}=6$ ). The mice had free access to water and standard rodent food pellets before and after surgery. Mice were excluded from the study if, during the surgical procedure to induce cerebral ischemia, (a) the occluding clamp on the common carotid artery (CCA) was in place for $\geq 5$ min (females: $n=2$ ), or (b) they died prior to $23.5 \mathrm{~h}$ reperfusion (males: $\mathrm{n}=4$; OVX: $\mathrm{n}=1)$.

\section{Ovariectomy of Female Mice}

Ovariectomy was performed in anesthetized $(80 \mathrm{mg} / \mathrm{kg}$ ketamine plus $10 \mathrm{mg} / \mathrm{kg}$ xylazine i.p.) 8- to 12 -week-old female $\mathrm{C} 57 \mathrm{Bl} / 6 \mathrm{~J}$ mice 7 days prior to cerebral ischemia, as previously described $[13,14]$. Animals were culled $24 \mathrm{~h}$ after stroke and brains were removed for analysis. To confirm the effectiveness of OVX surgery, the uterus was harvested and weighed (intact females: $123.5 \pm 22.0 \mathrm{mg}$; OVX: $76.1 \pm 51.0 \mathrm{mg}$ ).

\section{Focal Cerebral Ischemia}

Mice were randomly assigned to study groups (e.g. sham or stroke) by an investigator not performing surgical procedures or data analysis. Moreover, the investigator performing the surgical procedure or data analysis (e.g. neurological assessment, infarct volume quantification, immunohistochemistry) was usually (and wherever possible) blinded to the treatment group to which the animal or tissue belonged. Focal cerebral ischemia was produced by transient occlusion of the MCA [15-18]. Mice were anesthetized with ketamine $(80 \mathrm{mg} / \mathrm{kg}$ i.p.) plus xylazine $(10 \mathrm{mg} / \mathrm{kg}$ i.p. $)$ with rectal temperature monitored and maintained at $37.0 \pm$ $0.5^{\circ} \mathrm{C}$. The right carotid bifurcation was exposed through a ventral midline neck incision and the external carotid artery (ECA) ligated distal to the bifurcation of the CCA and cut, forming an ECA stump. The CCA was clamped and tension applied to the internal carotid artery (ICA) using a suture bridge. A small nick was made in the ECA stump, and a 6-0 nylon monofilament with silicone-coated tip inserted and advanced distally along the ICA (11-12 mm distal to the carotid bifurcation), causing MCA occlusion (MCAO) at its junction with the Circle of Willis. Severe $(\sim 75 \%)$ reduction in regional cerebral blood flow ( $\mathrm{rCBF}$ ) was confirmed using transcranial laser Doppler flowmetry (Perimed) in the area of cerebral cortex supplied by the MCA $(\sim 2 \mathrm{~mm}$ posterior and $\sim 5 \mathrm{~mm}$ lateral to bregma). The filament was then tied in place, the CCA clamp removed and the suture bridge released. Occlusion was maintained for $30 \mathrm{~min}$, and the filament retracted to allow reperfusion for $23.5 \mathrm{~h}$ (confirmed by an increase in $\mathrm{rCBF}$ ). Sham-operated mice were anesthetized and the right carotid bifurcation exposed, but no filament was inserted.

\section{Functional Impairment}

A hanging wire test was performed in which mice were suspended by their forelimbs on a wire stretched between two posts $30 \mathrm{~cm}$ above a foam pillow for up to $60 \mathrm{~s}[15,17,19]$. The time un- 
til the animal fell (a score of zero was assigned to animals that fell immediately and a score of 60 was assigned to animals that did not fall) was recorded and the average time of 3 trials with $5 \mathrm{~min}$ rest in between was calculated. This task is used as a measure of grasping ability and forelimb strength.

\section{Evaluation of Cerebral Infarct Volume}

Mice were killed at $24 \mathrm{~h}$ after cerebral ischemia by isoflurane inhalation, followed by decapitation. The brains were immediately removed, snap frozen in liquid nitrogen, and stored at $-80^{\circ} \mathrm{C}$. Evenly spread (separated by $\sim 420 \mu \mathrm{m}$ ) coronal sections (30 $\mu \mathrm{m}$ thick) were obtained spanning the infarct, thaw mounted onto poly-L-lysine-coated glass slides, and stained with thionin $(0.1 \%)$ to delineate the infarct. Images of the sections were captured with a CCD camera (Cohu Inc., San Diego, Calif., USA) mounted above a light box. Infarct volume was quantified using image analysis software (ImageJ, NIH), correcting for brain edema, according to the following formula: CIV $=[\mathrm{LHA}-(\mathrm{RHA}-$ RIA)] $\times$ (thickness of section + distance between sections); where CIV is corrected infarct volume, LHA is left hemisphere area, RHA is right hemisphere area and RIA is right hemisphere infarct area. Edema volume was calculated using the formula: [RHA LHA] $\times$ (thickness of section + distance between sections). Edema-corrected infarct volumes of individual brain sections were then added, giving a three dimensional approximation of the total infarct volume.

\section{GPER Immunohistochemistry}

GPER immunoreactivity in the brain was performed using methods similar to those reported previously [10]. Brains were removed and stored as described above. Multiple serial $10 \mu \mathrm{m}$ coronal sections that spanned the infarct (bregma $+0.84,+0.42$, $0,-0.42,-0.84,-1.26,-1.68$, and $-2.10 \mathrm{~mm}$ ) were taken for analysis. Brain sections were fixed in acetone and washed in $0.01 \mathrm{M}$ phosphate-buffered saline (PBS, $3 \times 10 \mathrm{~min}$ ). Ten percent goat serum was applied for $30 \mathrm{~min}$ and sections were then incubated in a GPR30 rabbit polyclonal antibody (1:200; Abcam) overnight in a humid box. For a negative control, the primary antibody was pre-incubated with the blocking peptide for $1 \mathrm{~h}$ before being applied to the tissue sections. The following day, tissues were washed in $0.01 \mathrm{M}$ PBS $(3 \times 10 \mathrm{~min})$ to remove excess antibody, and incubated in a Texas Red-conjugated goat anti-rabbit IgG (1:200; Zymed Laboratories) for $2-3 \mathrm{~h}$ in a humid box. Sections were then washed in PBS $(3 \times 5 \mathrm{~min})$, mounted and coverslipped.

Human brain tissue (see human case reports below) was obtained and fixed in 10\% neutralized formalin for a minimum of 2 weeks before being processed in paraffin wax, cut into slices and stored at $4^{\circ} \mathrm{C}$. GPER immunohistochemistry was performed on these sections using a similar process as described above. In addition, tissue sections were blocked with a peroxidase blocking agent for $10 \mathrm{~min}$ and then washed in PBS $(3 \times 10 \mathrm{~min})$ prior to adding $10 \%$ goat serum. Furthermore, the primary antibody was recognized using the DAKO EnVision + system (DAKO). Sections were incubated for $45 \mathrm{~min}$ with a peroxidase-labeled polymer conjugated to goat anti-rabbit IgG, washed in PBS $(3 \times 10 \mathrm{~min})$, followed by incubation with diaminobenzidine for $5 \mathrm{~min}$.

To identify in which cell type GPER was located, double-labeled immunofluorescence was performed on brain sections from sham and stroke mice. Unfortunately, we were unable to carry out these studies on human brain samples as we had limited access to this tissue. Brain sections were prepared as described above and simultaneously incubated with a GPR30 rabbit polyclonal antibody (1:200; Abcam) and either a NeuN mouse monoclonal antibody (1:1,000; Chemicon) or a von Willebrand factor (vWF) mouse monoclonal antibody (1:1,000; Abcam) overnight in a humid box. The following day, tissues were washed in $0.01 \mathrm{M}$ PBS $(3 \times 10 \mathrm{~min})$ and incubated in a Texas Red-conjugated goat anti-rabbit IgG (1:200; Zymed Laboratories) for $2-3 \mathrm{~h}$ in a humid box. Sections were then again washed in $0.01 \mathrm{M}$ PBS $(3 \times 5 \mathrm{~min})$ and incubated in a biotinylated anti-mouse IgG reagent for 10 min. Brain sections were then washed in $0.01 \mathrm{M}$ PBS $(3 \times 10 \mathrm{~min})$ and Fluorescein Avidin DCS (Vector Laboratories) was applied for $5 \mathrm{~min}$. Sections were washed in $0.01 \mathrm{M}$ PBS $(3 \times 10 \mathrm{~min})$ and coverslipped. All tissue-mounted slides used for immunohistochemistry were viewed and photographed with an Olympus fluorescence/light microscope.

GPER immunoreactivity was examined in 5 structures of mouse brain: the hippocampus at 3 levels from bregma -1.26 to $-2.1 \mathrm{~mm}$, the somatosensory cortex at 8 levels from +0.84 to $-2.1 \mathrm{~mm}$, the striatum at 5 levels from +0.84 to $-0.84 \mathrm{~mm}$, the thalamus at 3 levels from -1.26 to -2.10 , and the hypothalamus at 4 levels from bregma -0.84 to $-2.1 \mathrm{~mm}$. These structures were chosen because the infarct core (striatum and thalamus) and periinfarct zone (hippocampus, somatosensory cortex, and hypothalamus) are typically observed in these regions of the brain of the MCAO mouse model. GPER immunoreactivity in the mouse brain was observed at a magnification of $\times 200$ or $\times 400$ and each region was given a score based on the subjective judgment of two blinded observers. Human brain sections were examined at a magnification of $\times 4, \times 100, \times 200$, and $\times 400$. All the appropriate primary and secondary controls were performed.

\section{Western Blot Analysis}

Expression of GPER was measured in homogenates of the entire right (ischemic) cerebral hemisphere from mice following sham or stroke surgery using Western blotting. The right hemispheres were removed, snap frozen in liquid nitrogen and homogenized in Laemmli buffer. Protein concentration was determined using the reducing agent and detergent compatible (RC DC) protein assay. Equal amounts of protein were loaded onto a $10 \%$ polyacrylamide gel and transferred to a polyvinylidene membrane. Membranes were blocked with $5 \%$ skim milk in TBS$\mathrm{T}$ (Tris-buffered saline in $0.1 \%$ Tween 20 ) for $1 \mathrm{~h}$ and then incubated overnight $\left(4^{\circ} \mathrm{C}\right)$ with the appropriate primary antibody (GPER or $\beta$-actin) in 5\% skim milk and TBS-T. Membranes were then incubated for $1 \mathrm{~h}$ with a horseradish peroxidase-conjugated anti-rabbit IgG for GPER and HRP-conjugated anti-mouse IgG for $\beta$-actin. Immunoreactive bands were detected by enhanced chemiluminescence, quantified using a ChemiDoc XRS molecular imager and normalized to the intensity of corresponding bands for $\beta$-actin.

\section{Quantitative Real-Time PCR}

Total RNA was isolated from the entire right hemisphere from male mice using the RNeasy Mini Kit with oncolumn DNase step (Qiagen, Hilden, Germany) followed by cDNA conversion using the Quantitect Reverse transcription kit (for Taqman ${ }^{\circledR}$ gene expression assays; Qiagen). We did not examine the right cerebral hemisphere from intact female or OVX mice because the immu- 
nohistochemical and Western blot data indicated that stroke had little or no effect on GPER expression in the female brain, which was in contrast to males. Pre-designed Taqman gene expression assays were then used to specifically measure mRNA expression of GPER (Mn01194815; NCBI Reference Sequence: RefSeq NM_029771.3), ER $\alpha$ (Mn01191130; RefSeq NM_007956.4) and ERß (Mn00599820; RefSeq NM_NM010157.3), and $\beta$-actin (Mn4352341; RefSeq NM_007393.1; Applied Biosystems, Carlsbad, Calif., USA) as a house-keeping gene. Assays were performed according to the manufacturer's instructions using the Bio-Rad CFX96TM real-time PCR machine (Bio-Rad, Hercules, Calif., USA). Data were normalized to the housekeeping gene and calculated as changes in fold expression relative to sham using the formula: fold change $=2^{-\Delta \Delta C T}$.

\section{Human Case Reports}

Brain tissue was obtained from stroke patients at National Taiwan University Hospital and the study was approved by the National Taiwan University Hospital ethics committee.

Patient 1. This 40 -year-old man was diagnosed with pancytopenia, with a bone marrow biopsy suggesting severe aplastic anemia. There was a poor response to treatment, which included bone marrow transplantation, and the patient died due to repeated severe infections and associated cardiac and respiratory failure.

Patient 2. This 1-year-old girl had decreased oral intake, weight loss, and was pale for several months. Distended abdomen with severe hepatosplenomegaly was noted and cytomegalovirus infection was suspected after liver and spleen biopsies. Ganciclovir and Cytotec were given but the patient died due to progressive hepatosplenomegaly and associated multiple medical complications.

Patient 3. A 39-year-old man had an acute brainstem stroke and died after 9 days with massive infarcts and obstructive hydrocephalus. Acute basilar artery occlusion related to atherosclerosis and associated thrombi were suggested as possible causes of death on autopsy.

Patient 4. This 45-year-old female had sudden loss of consciousness and CT revealed acute subarachnoid hemorrhage and intracerebral hemorrhage in the left frontal area. Angiography showed an anterior communicating artery aneurysm and operation was performed. Follow-up CT 10 days later showed acute infarct in the left anterior cerebral artery territory. The patient then suffered from severe sepsis and gastroenteric paralysis, leading to multiple organ failure and disseminated intravascular coagulation and the patient died 22 days after stroke onset.

\section{Statistical Analysis}

All data are presented as mean \pm standard error. Statistical analyses were performed using GraphPad Prism version 5 (GraphPad Software Inc., San Diego, Calif., USA). Between-group comparisons were compared using one-way ANOVA, a Student's unpaired t test or a one sample t test, as appropriate. If differences were detected by ANOVA, individual groups were compared with the Student-Newman-Keuls test. Statistical significance was accepted when $\mathrm{p}<0.05$.

\section{Results}

rCBF decreased to $\sim 20-30 \%$ of the pre-ischemia level for the duration of MCAO in males, intact females, and OVX mice (fig. 1a). rCBF returned to $\sim 100 \%$ within 5 min of the withdrawal of the monofilament, and then stabilized between 80 and $90 \%$ after 30 min of reperfusion. Mortality rate following ischemia reperfusion was similar between males, intact females, and OVX females (5-10\%; data not shown). Hanging wire times were significantly reduced in males and OVX mice versus intact females (fig. 1b). Consistent with hanging wire outcomes, cerebral infarct volume was significantly larger in males versus intact females and appeared increased, but was not significantly different in OVX versus intact female mice (fig. 1c, d). There was no significant difference in edema values between groups (data not shown).

\section{Localization of GPER in the Brain after Stroke}

A specific anti-GPER antibody was used to identify the distribution of GPER in the mouse brain after sham or stroke surgery. The GPER immunoreactivity data presented are semi-quantitative assessments based on subjective judgment of two independent observers. Moderate GPER immunoreactivity was observed in most areas of the brain examined in sham animals of both sexes (fig. 2, table 1). However, high immunoreactivity was present in the striatum and thalamus of males and females, whereas low GPER immunoreactivity was observed in the hypothalamus of OVX mice. The pattern of GPER immunoreactivity indicated that GPER was localized in neurons and cerebral capillaries. This was confirmed using double-labeling immunohistochemistry, which revealed GPER immunofluorescence co-localized with the neuronal marker, NeuN, and the endothelial cell marker, vWF (fig. 3). Importantly, very weak or no immunoreactivity was present in sections incubated with the GPER antibody pre-absorbed with the GPER blocking peptide, consistent with specific immunostaining (fig. 3h).

In male mice $24 \mathrm{~h}$ after stroke, GPER immunoreactivity was increased in all 3 peri-infarct regions: hippocampus, somatosensory cortex, and hypothalamus (fig. 2, table 1). In contrast, GPER immunoreactivity was decreased in the striatum and thalamus following stroke (fig. 2, table 1). Furthermore, GPER immunoreactive neurons in the peri-infarct regions of the male brain after stroke appeared to be more intensely labeled in comparison to sham controls. In brains of intact females assessed $24 \mathrm{~h}$ after stroke, GPER immunoreactivity was decreased in 


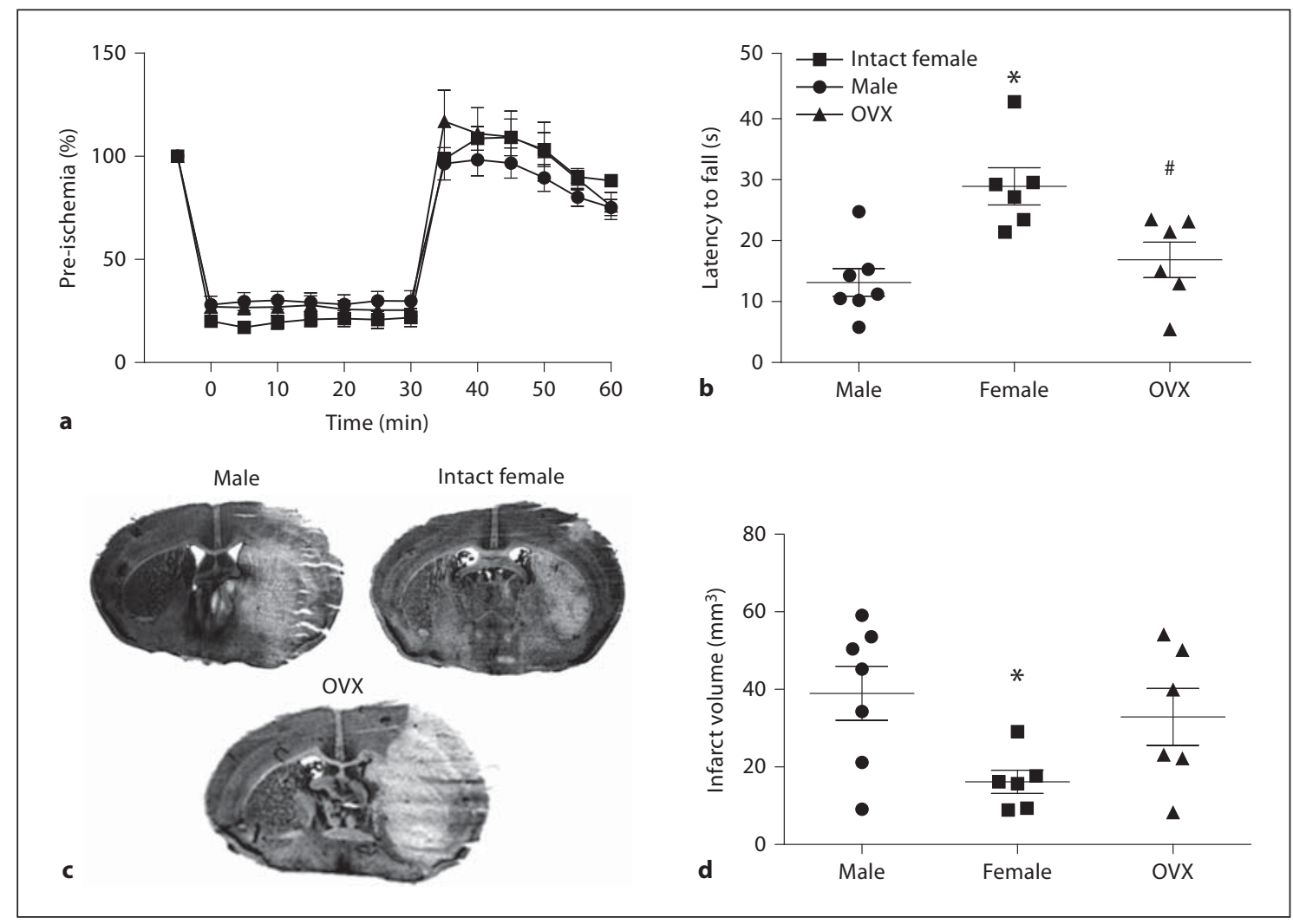

Fig. 1. $\mathrm{rCBF}$, infarct volume and hanging wire scores at $24 \mathrm{~h}$ in male, female, and OVX mice. a rCBF was recorded during and after 30-min MCAO with reperfusion. Hanging wire scores (b), representative thioninstained coronal brain sections (c), and cerebral infarct volumes (d) are shown from male, intact female, and OVX mice after MCAO. Data are presented as mean \pm SEM. ${ }^{*} \mathrm{p}<0.05$ vs. male; ${ }^{*} \mathrm{p}<0.05$ vs. intact female.

Table 1. Semi-quantitative analysis of the distribution of GPER immunoreactivity in the brain of male $(n=7)$, female $(n=6)$, and OVX $(n=6)$ mice 24 h after sham or stroke surgery

\begin{tabular}{|c|c|c|c|c|c|c|c|}
\hline & \multirow[t]{2}{*}{ Brain region } & \multicolumn{2}{|l|}{ Male } & \multicolumn{2}{|c|}{ Female } & \multicolumn{2}{|l|}{ OVX } \\
\hline & & sham & stroke & sham & stroke & sham & stroke \\
\hline \multirow{6}{*}{ Peri-infarct } & Hippocampus & & & & & & \\
\hline & CA1 & ++ & +++ & + & + & + & + \\
\hline & CA2 & + & ++ & ++ & + & ++ & + \\
\hline & CA3 & ++ & +++ & ++ & + & ++ & ++ \\
\hline & Somatosensory cortex & ++ & +++ & ++ & ++ & ++ & ++ \\
\hline & Hypothalamus & ++ & +++ & ++ & ++ & + & + \\
\hline \multirow[t]{2}{*}{ Infarct } & Striatum & +++ & ++ & +++ & ++ & ++ & + \\
\hline & Thalamus & +++ & ++ & +++ & ++ & ++ & + \\
\hline
\end{tabular}

Intensity of label: $+=$ low; $++=$ moderate $+++=$ high. 


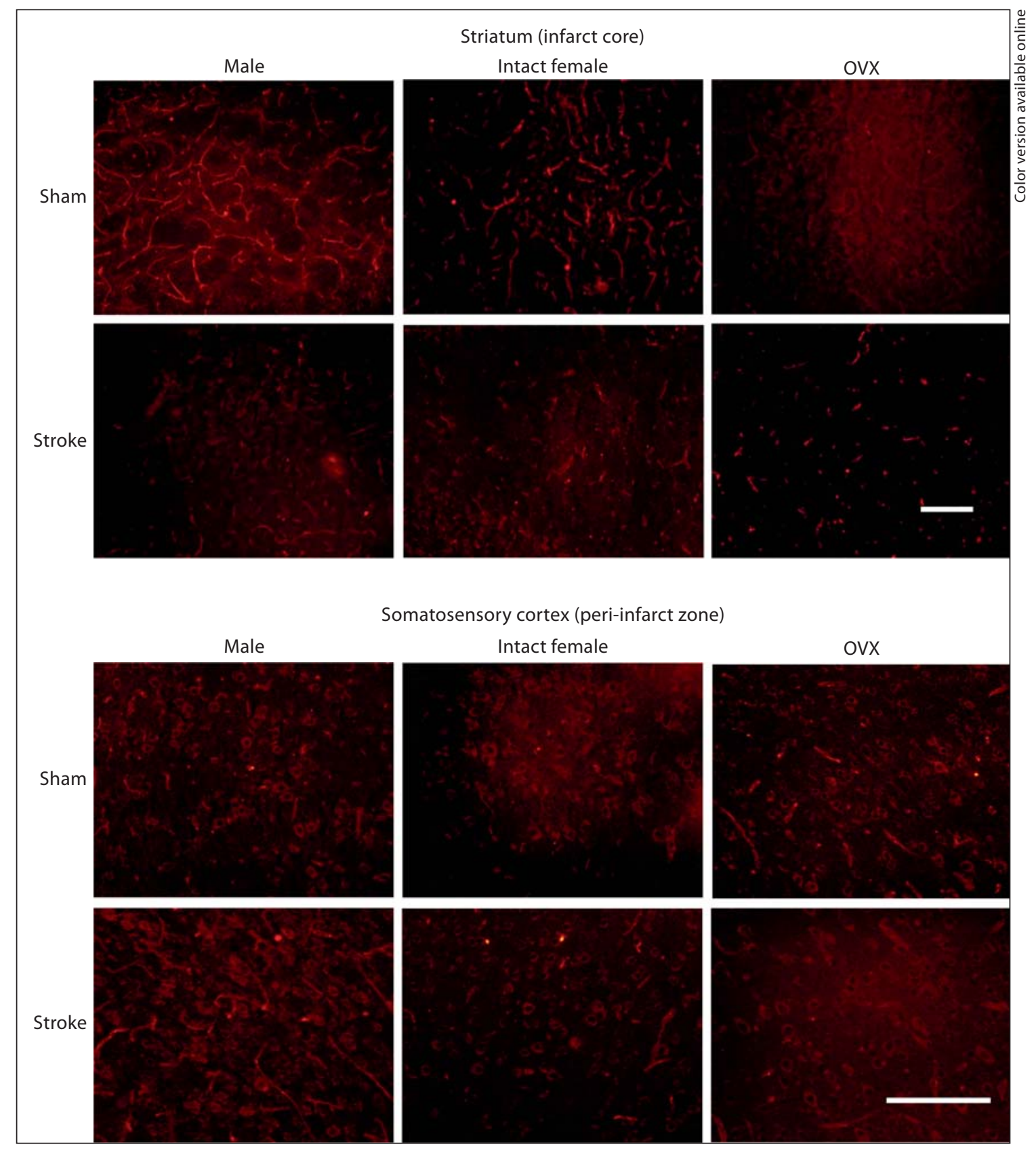

Fig. 2. Representative photomicrographs showing GPER immunoreactivity in the infarct (striatum) and periinfarct (somatosensory cortex) regions of the brain from male $(n=7)$, female $(n=6)$, and OVX $(n=6)$ mice $24 \mathrm{~h}$ after sham or stroke surgery. Scale bar $=100 \mu \mathrm{m}$.

the hippocampus, striatum, and thalamus, but not in the hypothalamus (fig. 2, table 1). In addition, less GPER immunoreactivity was observed in the striatum, thalamus, and the CA2 region of the hippocampus in OVX mice after stroke, but GPER distribution was unchanged in the somatosensory cortex and hypothalamus (fig. 2, table 1).
GPER immunoreactivity was also assessed post mortem in 2 male and 2 female human brains - one non-stroke and one stroke patient per sex - and these studies provided the first anatomical evidence that GPER is expressed in the human brain (fig. 4). These data are obviously preliminary, qualitative and uncontrolled, and our observations 

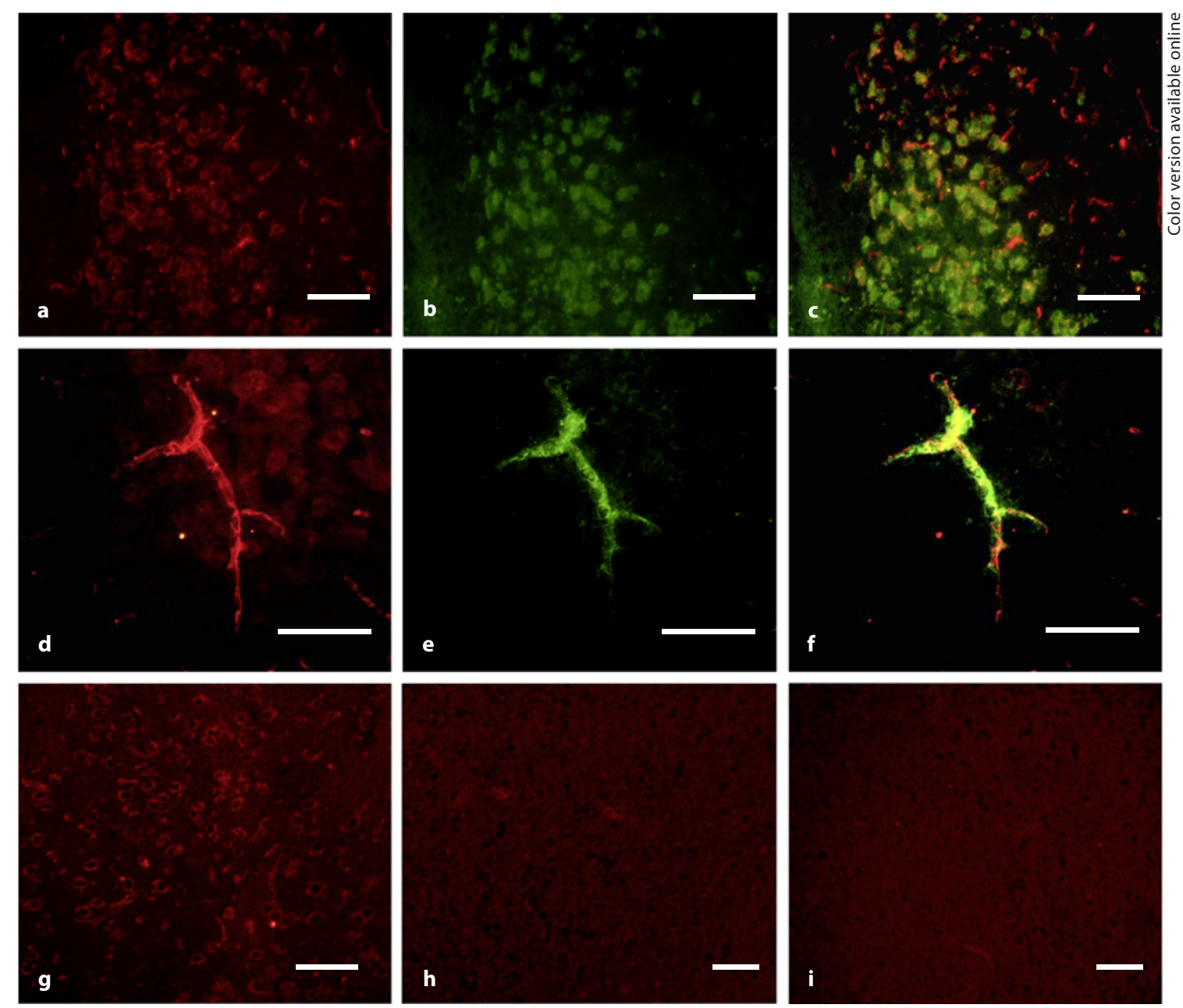

Fig. 3. GPER is located in neurons and the cerebral microvasculature. Representative photomicrographs showing GPER (a, d, g), NeuN (b) or vWF (e) in the brain from male mice. Overlayed images revealing GPER is co-localized with NeuN (c) and vWF (f). Control sections showing very faint or no GPER immunoreactivity following the addition of the GPER blocking peptide (h) or the removal of the GPER antibody (i). Scale bar $=50 \mu \mathrm{m}$.

require careful follow-up via group data. Nevertheless, consistent with the immunohistochemical findings in mice, GPER immunostaining in the ischemic hemisphere appeared to be higher in the post-stroke male brain compared with the control male, whereas little difference in immunoreactivity was noted between the female patients (fig. 4). In contrast to mice, however, no GPER immunoreactivity was observed in the cerebrovasculature.

\section{Protein Expression and $m R N A$ Levels of GPER in the}

Brain after Stroke

We examined protein expression of GPER in the mouse brain following stroke, as well as mRNA levels of GPER and the traditional estrogen receptors, ER $\alpha$ and ER $\beta$.
Supporting the immunohistochemical observations, we found that GPER protein expression as measured by Western blotting was increased in male brains $24 \mathrm{~h}$ after stroke (fig. 5a, b). Conversely, GPER expression was not different between brains from intact female and OVX mice $24 \mathrm{~h}$ after stroke or sham stroke surgery (fig. $5 \mathrm{a}, \mathrm{c}, \mathrm{d}$ ). These findings are consistent with our separate immunohistochemical analyses of most brain regions examined in intact female and OVX mice following stroke. Furthermore, mRNA expression of GPER in the ischemic hemisphere of male mouse brains was elevated at $4 \mathrm{~h}$ after stroke $(\mathrm{p}<$ 0.05 ), but had returned to the baseline level by $24 \mathrm{~h}$ (fig. $6 \mathrm{a}$ ). Unlike GPER, however, ER $\alpha$ and ER $\beta$ mRNA expression at $4 \mathrm{~h}$ after stroke was not significantly altered (fig. 6b, c). 
Fig. 4. Photomicrographs showing GPER immunoreactivity (arrows) in human brain sections from non-stroke (patient 1 , male; patient 2, female) or stroke (patient 3 , male; patient 4, female) individuals. Magnification $\times 200$ (inset $\times 400$ ).

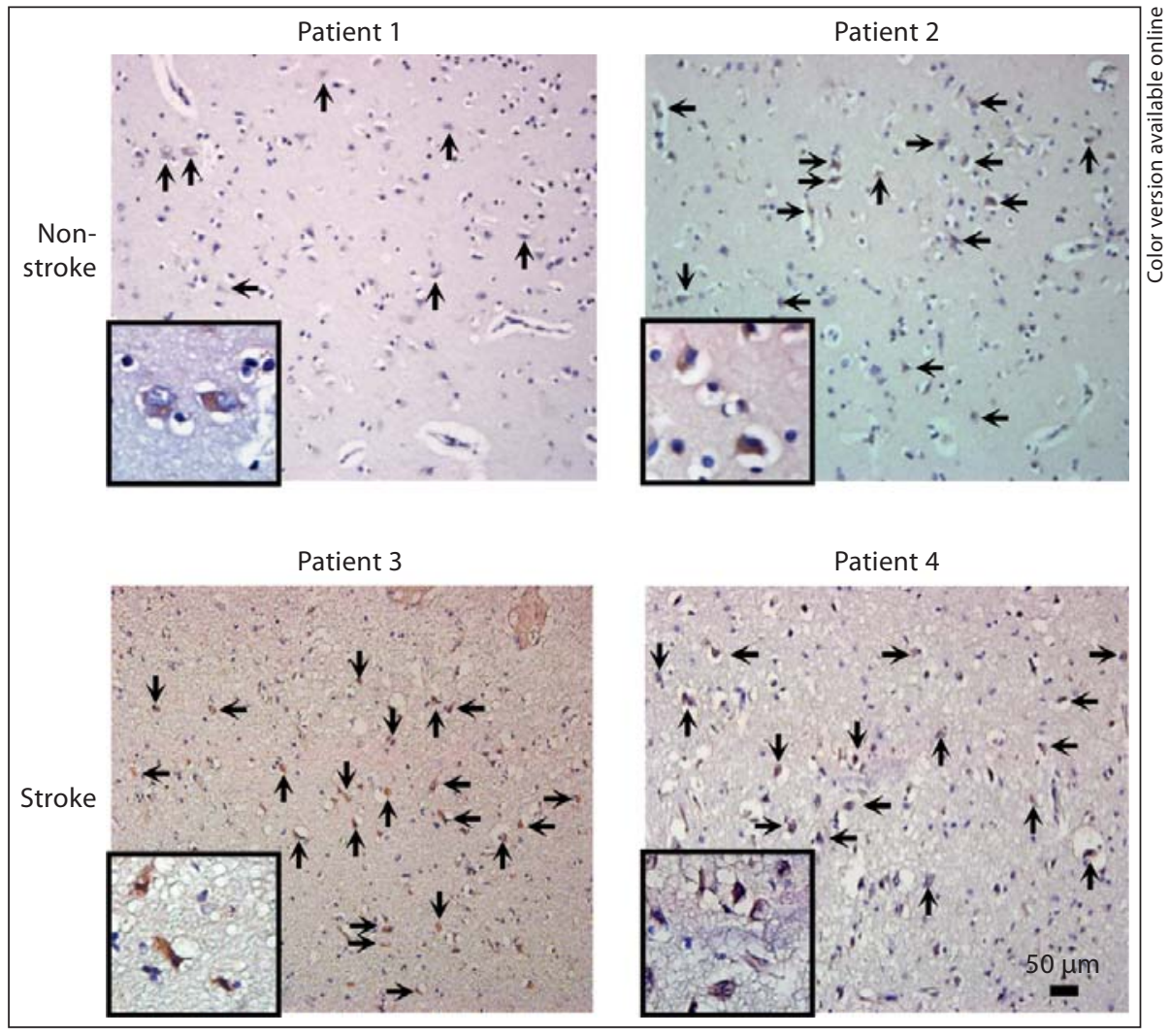

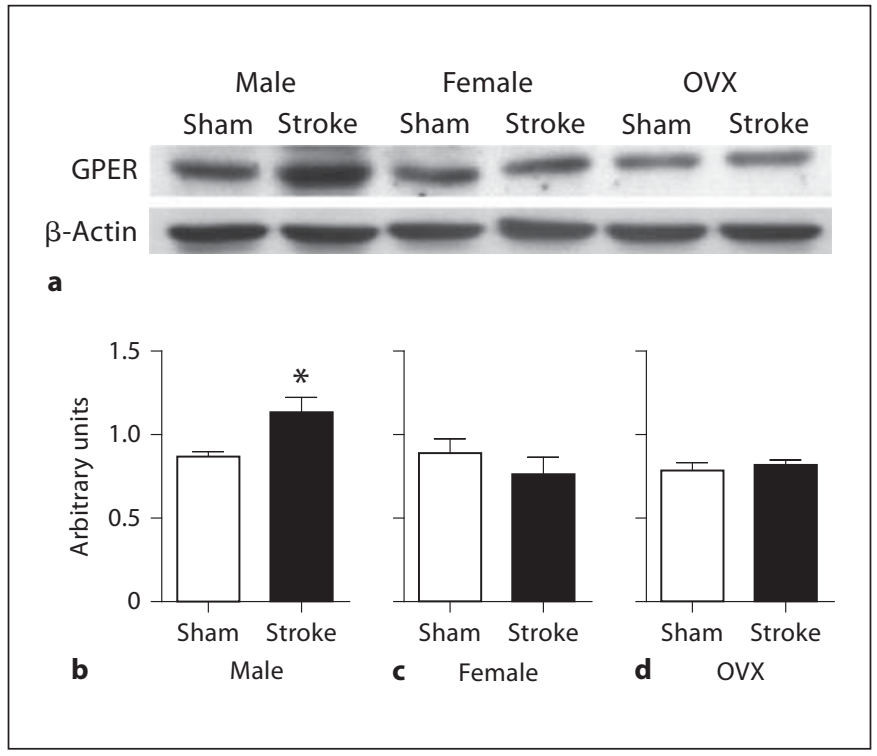

Fig. 5. a Representative Western blot showing GPER protein expression in the ischemic cerebral hemisphere of male, female, and OVX mice. $\mathbf{b}-\mathbf{d}$ Immunoblot analysis of GPER band intensities revealed that stroke increased GPER levels in male brains $(n=5)$ but had little or no effect in female $(n=5)$ and OVX $(n=5)$ brains. Data are presented as mean \pm SEM. ${ }^{*} \mathrm{p}<0.05$ vs. sham.

\section{Discussion}

This is the first study to assess GPER distribution and expression in the brain after stroke. The findings indicate that in the male brain GPER distribution and expression is increased following stroke, whereas little or no change occurs in intact or OVX females. In addition, preliminary data indicate that GPER is expressed in the human brain and its distribution may be greater in the male but not female after stroke. Our studies in mice further demonstrated that GPER is localized in both neurons and the cerebral microvasculature. Overall, the findings from this study suggest that stroke differentially affects GPER expression and distribution in the brain of males and females (whether intact or OVX), and could thus play a sexspecific role in stroke outcome.

In recent years, it has become apparent that GPER plays a prominent role in multiple aspects of the central nervous system [20]. Except for a few select regions in the brain [21], GPER distribution is generally distinct from that of classical estrogen receptors, and in fact, appears to be more highly expressed [6]. Previous studies by Brailoiu et al. [9] and Hazell et al. [6] demonstrated that GPER 
Fig. 6. Time-dependent expression of estrogen receptors in the mouse brain after cerebral ischemia reperfusion. Data from Taqman assays showing expression of mRNA for GPER (a), ER $\alpha$ (b), and ER $\beta$ (c) in ischemic hemisphere after $0.5 \mathrm{~h}$ ischemia plus 4 or $24 \mathrm{~h}$ reperfusion relative to sham ( $n=3$ per group). Data are presented as mean \pm SEM. ${ }^{*} \mathrm{p}<0.05$ vs. sham.

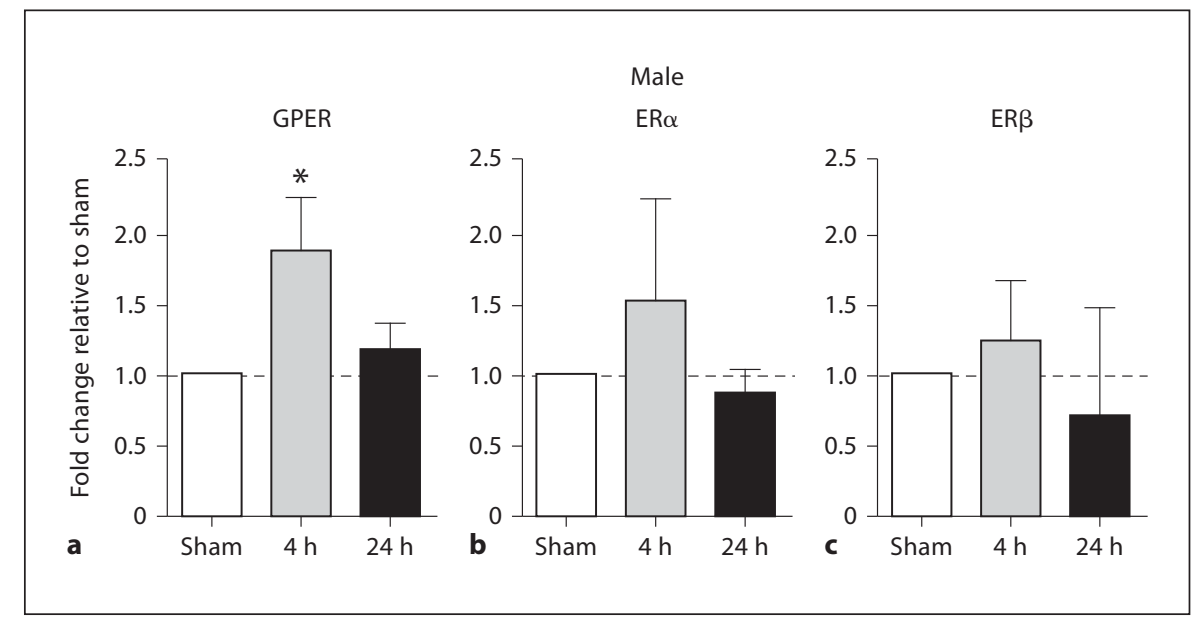

distribution is moderate to high within many areas of the brain, including the hippocampus, somatosensory cortex, thalamus, striatum, and hypothalamus. In addition, both studies reported that GPER distribution in the rat $[6,9]$ and mouse [6] brain is comparable between sexes. Consistent with this, the current findings demonstrate a moderate level of GPER immunoreactivity in the hippocampus, somatosensory cortex, and hypothalamus of male and female control mice. Moreover, a high level of GPER immunoreactivity was observed in the striatum and thalamus in both sexes. These findings are, however, in contrast to those of Canonaco et al. [22] who reported that a sexually dimorphic GPER expression pattern occurs in the hypothalamus and thalamus of the hamster. Nevertheless, there is accumulating evidence that GPER distribution in the brain is typically quite similar between males and females. A moderate level of GPER immunoreactivity was also observed in the hippocampus and somatosensory cortex of OVX mice following sham surgery. Unlike males and intact females, however, only a moderate level of GPER immunoreactivity was detected in the striatum and a low level of GPER was distributed in the hypothalamus. This latter finding is consistent with recent observations by Spary et al. [8] who showed that the number of GPER-positive cells in the paraventricular nucleus, a discrete band of nerve cells in the anterior part of the hypothalamus, is decreased in females following OVX. Thus, it seems that estrogen may have a greater effect on GPER expression in some brain regions compared to others.

Immunohistochemical studies have identified that GPER is not predominantly expressed in any one neuronal phenotype. For example, GPER is co-localized with nitrergic neurons, TH-positive catecholaminergic neurons, ChAT-positive cholinergic neurons, 5-HT-positive serotonergic neurons, and oxytocin and vasopressin neurons [8]. In the current study, the pattern of GPER-positive immunoreactivity appeared to be located in neurons and the microvasculature. To confirm that GPER was expressed in neurons, we performed double-labeled immunohistochemistry using the GPER antibody and the neuronal cell marker, NeuN. GPER immunoreactivity was co-localized with NeuN, thus verifying that GPER is located in the neuronal soma (cell body). GPER immunoreactivity appeared to be located in the neuronal cytoplasm, most likely in the endoplasmic reticulum and/or Golgi apparatus, as widely reported $[1,8,9,23,24]$. In addition, the current study showed for the first time that GPER immunoreactivity is also co-localized with the endothelial cell marker, vWF. This indicates that GPER is expressed in the endothelium of the cerebral microvasculature. This observation in small parenchymal vessels is consistent with our finding that GPER is present in both the endothelium and vascular smooth muscle of the rat CCA and MCA [10]. It is worth noting that in some small cerebral arterioles, the orientation of GPER immunoreactivity suggested that it was also located in the smooth muscle. It is tempting to speculate that since GPER mediates cerebral vasodilatation, as reported by our group [10] and by others [25], perhaps GPER signaling in cerebral vessels improves blood flow to the infarct and penumbra region, thereby providing neuroprotection following stroke.

Due to its high expression and widespread distribution in the brain and cerebral vasculature, GPER is a potential therapeutic target for stroke. In fact, preliminary 
studies by Zhang et al. [11] and Lebesgue et al. [12] demonstrated that the GPER agonist, G-1, reduces stroke-induced brain damage in OVX rodents. However, it remained to be determined as to whether GPER distribution and expression in the brain is increased following stroke. The present study found that GPER distribution in the brain is increased in male mice after stroke. More specifically, GPER distribution in the hippocampus, somatosensory cortex, and hypothalamus is increased in mice subjected to stroke. It is these brain regions that typically correspond with the peri-infarct zone of the mouse model of stroke. In contrast, stroke did not alter GPER distribution in the striatum, and it was decreased in the thalamus. A likely explanation for the lack of change in GPER distribution in the striatum and thalamus is that neuronal loss is greatest in these two areas following stroke. Consistent with these findings in the peri-infarct regions of the brain, Western blot analysis revealed that GPER protein expression is selectively increased in the ipsilateral hemisphere of male mice. Furthermore, GPER mRNA levels were also found to be elevated in the male brain at $4 \mathrm{~h}$ after stroke. Interestingly however, ER $\alpha$ mRNA levels in the male brain were not altered after stroke. This finding correlates with observations by Westberry et al. [26], who reported that ER $\alpha$ mRNA levels do not change in the ischemic cortex of male rats.

It is becoming increasingly recognized that stroke is a sexually dimorphic disease. For example, ER $\alpha$ mRNA expression in the ischemic cortex of male rodents is not altered following stroke [26, 27]. In contrast, several studies have reported that ER $\alpha$ mRNA expression is increased in the ischemic cortex of vehicle- and estradiol-treated OVX female rodents [26-28], which indicates that ER $\alpha$ levels are elevated following stroke in females independent of the level of circulating estrogen. Conversely, the current study found that GPER immunoreactivity in the female brain did not change following stroke, except in the hippocampus, in which distribution was decreased. Similarly, GPER distribution in the OVX brain was either not altered (somatosensory cortex, hypothalamus) or reduced (hippocampus, striatum, and thalamus) following ischemia. In the male brain, however, GPER distribution was increased in the hippocampus, somatosensory cortex, and the hypothalamus. These findings were supported by Western blot analysis showing that GPER protein expression in the ischemic hemisphere is only elevated in males. Together these findings suggest that GPER distribution and expression in the male, but not the intact female or OVX brain, is increased following stroke. Collectively, increased GPER and ER $\alpha$ expression in the isch- emic hemisphere of males and females, respectively, suggests that these estrogen receptors could play sex-specific roles following stroke.

In the human brain, GPER has been reported to be highly expressed in the primary olfactory cortex, olfactory tubercle, nucleus of the lateral olfactory tract, hippocampal areas of Ammon's horn and dentate gyrus [29]. Furthermore, GPER mRNA was found to be densely distributed in the hypothalamic nuclei, such as the paraventricular, supraoptic, arcuate, and suprachiasmatic nuclei [29]. However, the current study is the first to examine GPER localization in the human brain after stroke. Our preliminary data suggest that GPER distribution may be increased in the male human brain following stroke.

In summary, the present study provides evidence that GPER distribution is increased in mouse brain regions corresponding with the peri-infarct zone of our MCAO model of stroke, in males but not intact female or OVX brains. These findings shed further light on the highly complex estrogen signaling cascade in male and female brains following stroke. Overall, GPER is potentially an important therapeutic target following stroke, especially in males, in whom estrogen therapy is not feasible.

\section{Acknowledgments}

We would like to thank Yu-Hsuan Hsieh for her technical assistance. These studies were supported by a project grant from the National Health and Medical Research Council of Australia (NHMRC; ID 491133). B.R.S.B. was supported by a postdoctoral fellowship from the National Heart Foundation of Australia. V.H.B. was supported by a Monash graduate scholarship. T.V.A. was supported by an Australian research council future fellowship. A.A.M. is a career development award fellow of the NHMRC. G.R.D. and C.G.S. are senior research fellows of the NHMRC.

References terburn JB, Prossnitz ER: A transmembrane intracellular estrogen receptor mediates rapid cell signaling. Science 2005;307:16251630.

2 Thomas P, Pang Y, Filardo EJ, Dong J: Identity of an estrogen membrane receptor coupled to a $\mathrm{G}$ protein in human breast cancer cells. Endocrinology 2005;146:624-632.

- 3 Prossnitz ER, Arterburn JB, Smith HO, Oprea TI, Sklar LA, Hathaway HJ: Estrogen signaling through the transmembrane $G$ protein-coupled receptor GPR30. Annu Rev Physiol 2008;70:165-190.

-4 Olde B, Leeb-Lundberg LM: GPR30/GPER1: searching for a role in estrogen physiology. Trends Endocrinol Metab 2009;20:409-416. 
5 Isensee J, Meoli L, Zazzu V, Nabzdyk C, Witt H, Soewarto D, Effertz K, Fuchs H, GailusDurner V, Busch D, Adler T, de Angelis MH, Irgang $\mathrm{M}$, Otto $\mathrm{C}$, Noppinger PR: Expression pattern of $\mathrm{G}$ protein-coupled receptor 30 in lacz reporter mice. Endocrinology 2009;150: 1722-1730.

-6 Hazell GG, Yao ST, Roper JA, Prossnitz ER, O'Carroll AM, Lolait SJ: Localisation of GPR30, a novel G protein-coupled oestrogen receptor, suggests multiple functions in rodent brain and peripheral tissues. J Endocrinol 2009;202:223-236.

-7 Martensson UE, Salehi SA, Windahl S, Gomez MF, Sward K, Daszkiewicz-Nilsson J, Wendt A, Andersson N, Hellstrand P, Grande PO, Owman C, Rosen CJ, Adamo ML, Lundquist I, Rorsman P, Nilsson BO, Ohlsson C, Olde B, Leeb-Lundberg LM: Deletion of the $\mathrm{G}$ protein-coupled receptor 30 impairs glucose tolerance, reduces bone growth, increases blood pressure, and eliminates estradiol-stimulated insulin release in female mice. Endocrinology 2009;150:687698.

-8 Spary EJ, Chapman SE, Sinfield JK, Maqbool A, Kaye J, Batten TF: The novel G proteincoupled oestrogen receptor GPR30 shows changes in $\mathrm{mRNA}$ expression in the rat brain over the oestrous cycle. Neurosignals 2012, DOI: 10.1159/000333296.

-9 Brailoiu E, Dun SL, Brailoiu GC, Mizuo K, Sklar LA, Oprea TI, Prossnitz ER, Dun NJ: Distribution and characterization of estrogen receptor $G$ protein-coupled receptor 30 in the rat central nervous system. J Endocrinol 2007;193:311-321.

10 Broughton BR, Miller AA, Sobey CG: Endothelium-dependent relaxation by $G$ protein coupled receptor 30 agonists in rat carotid arteries. Am J Physiol Heart Circ Physiol 2010;298:1055-1061.

-11 Zhang B, Subramanian S, Dziennis S, Jia J, Uchida M, Akiyoshi K, Migliati E, Lewis AD, Vandenbark AA, Offner H, Hurn PD: Estradiol and G1 reduce infarct size and improve immunosuppression after experimental stroke. J Immunol 2010;184:4087-4094.
12 Lebesgue D, Traub M, De Butte-Smith M, Chen C, Zukin RS, Kelly MJ, Etgen AM: Acute administration of non-classical estrogen receptor agonists attenuates ischemiainduced hippocampal neuron loss in middle-aged female rats. PLoS One 2010;5:e8642.

13 Miller AA, Drummond GR, Mast AE, Schmidt HH, Sobey CG: Effect of gender on $\mathrm{NADPH}$-oxidase activity, expression, and function in the cerebral circulation: role of estrogen. Stroke 2007;38:2142-2149.

14 Chrissobolis S, Sobey CG: Influence of gender on $\mathrm{K}^{+}$-induced cerebral vasodilatation. Stroke 2004;35:747-752.

15 Brait VH, Rivera J, Broughton BR, Lee S, Drummond GR, Sobey CG: Chemokine-related gene expression in the brain following ischemic stroke: no role for CXCR2 in outcome. Brain Res 2011;1372:169-179.

16 Jackman KA, Miller AA, De Silva TM, Crack PJ, Drummond GR, Sobey CG: Reduction of cerebral infarct volume by apocynin requires pretreatment and is absent in Nox2deficient mice. Br J Pharmacol 2009;156: 680-688.

17 Brait VH, Jackman KA, Walduck AK, Selemidis S, Diep H, Mast AE, Guida E, Broughton BR, Drummond GR, Sobey CG: Mechanisms contributing to cerebral infarct size after stroke: gender, reperfusion, $\mathrm{T}$ lymphocytes, and Nox2-derived superoxide. J Cereb Blood Flow Metab 2010;30:1306-1317.

18 Jackman KA, Miller AA, Drummond GR, Sobey CG: Importance of Nox1 for angiotensin II-induced cerebrovascular superoxide production and cortical infarct volume following ischemic stroke. Brain Res 2009; 1286:215-220.

19 Hattori K, Lee H, Hurn PD, Crain BJ, Traystman RJ, DeVries AC: Cognitive deficits after focal cerebral ischemia in mice. Stroke 2000; 31:1939-1944.

20 Prossnitz ER, Barton M: Signaling, physiological functions and clinical relevance of the $G$ protein-coupled estrogen receptor GPER. Prostaglandins Other Lipid Mediat 2009;89:89-97.

-21 Spary EJ, Maqbool A, Batten TF: Oestrogen receptors in the central nervous system and evidence for their role in the control of cardiovascular function. J Chem Neuroanat 2009;38:185-196.
2 Canonaco M, Giusi G, Madeo A, Facciolo RM, Lappano R, Canonaco A, Maggiolini M: A sexually dimorphic distribution pattern of the novel estrogen receptor G-protein-coupled receptor 30 in some brain areas of the hamster. J Endocrinol 2008;196:131-138.

23 Matsuda K, Sakamoto H, Mori H, Hosokawa K, Kawamura A, Itose M, Nishi M, Prossnitz ER, Kawata M: Expression and intracellular distribution of the $G$ protein-coupled receptor 30 in rat hippocampal formation. Neurosci Lett 2008;441:94-99.

24 Otto C, Rohde-Schulz B, Schwarz G, Fuchs I, Klewer M, Brittain D, Langer G, Bader B, Prelle K, Nubbemeyer R, Fritzemeier KH: G protein-coupled receptor 30 localizes to the endoplasmic reticulum and is not activated by estradiol. Endocrinology 2008; 149:48464856.

25 Patkar S, Farr TD, Cooper E, Dowell FJ, Carswell HV: Differential vasoactive effects of oestrogen, oestrogen receptor agonists and selective oestrogen receptor modulators in rat middle cerebral artery. Neurosci Res 2011;71:78-84

26 Westberry JM, Prewitt AK, Wilson ME: Epigenetic regulation of the estrogen receptor alpha promoter in the cerebral cortex following ischemia in male and female rats. Neuroscience 2008;152:982-989.

27 Elzer JG, Muhammad S, Wintermantel TM, Regnier-Vigouroux A, Ludwig J, Schutz G, Schwaninger M: Neuronal estrogen receptor-alpha mediates neuroprotection by $17 \mathrm{be}$ ta-estradiol. J Cereb Blood Flow Metab 2009; 30:935-942.

28 Dubal DB, Rau SW, Shughrue PJ, Zhu H, Yu J, Cashion AB, Suzuki S, Gerhold LM, Bottner MB, Dubal SB, Merchanthaler I, Kindy MS, Wise PM: Differential modulation of estrogen receptors (ERs) in ischemic brain injury: a role for ERalpha in estradiolmediated protection against delayed cell death. Endocrinology 2006;147:3076-3084.

29 O’Dowd BF, Nguyen T, Marchese A, Cheng R, Lynch KR, Heng HH, Kolakowski LF Jr George SR: Discovery of three novel G-protein-coupled receptor genes. Genomics 1998; 47:310-313. 